\title{
Pose Estimation for Mobile Robots to Maximise Data Quality of Fixed-Focus Laser Diagnostics in Hazardous Environments
}

DOI:

10.1109/IROS.2018.8593367

\section{Document Version}

Accepted author manuscript

Link to publication record in Manchester Research Explorer

Citation for published version (APA):

West, A., Watson, S., \& Lennox, B. (Accepted/In press). Pose Estimation for Mobile Robots to Maximise Data Quality of Fixed-Focus Laser Diagnostics in Hazardous Environments. Paper presented at IEEE International Conference on Intelligent Robots and Systems (IROS) 2018, Madrid, Spain.

https://doi.org/10.1109/IROS.2018.8593367

\section{Citing this paper}

Please note that where the full-text provided on Manchester Research Explorer is the Author Accepted Manuscript or Proof version this may differ from the final Published version. If citing, it is advised that you check and use the publisher's definitive version.

\section{General rights}

Copyright and moral rights for the publications made accessible in the Research Explorer are retained by the authors and/or other copyright owners and it is a condition of accessing publications that users recognise and abide by the legal requirements associated with these rights.

\section{Takedown policy}

If you believe that this document breaches copyright please refer to the University of Manchester's Takedown Procedures [http://man.ac.uk/04Y6Bo] or contact uml.scholarlycommunications@manchester.ac.uk providing relevant details, so we can investigate your claim.

\section{OPEN ACCESS}




\title{
Pose Estimation for Mobile Robots to Maximise Data Quality of Fixed-Focus Laser Diagnostics in Hazardous Environments
}

\author{
Andrew West, Simon Watson and Barry Lennox
}

\begin{abstract}
Characterisation of nuclear environments is critical for long term operation and decommissioning. Laser Induced Breakdown Spectroscopy (LIBS) is an example of a scientific instrument that could be deployed to aid in characterisation of unknown environments. LIBS consists of a high intensity pulsed laser being focussed down onto a target to create a plasma, and optical emission from the plasma is then used to determine elemental composition of unknown materials. For robots deployed with these instruments in extreme environments, mission time can be limited by hazards present such as radiation. Once deployed a robot must be able to collect the best data possible whilst maximising operational runtime. We present a data quality based probabilistic approach to robot pose estimation to maximise data quality, by considering optimum sensor placement whilst avoiding harmful environmental features such as radiation for a fixed-focus laser diagnostic such as LIBS. This approach is able to determine optimum robot poses for arbitrary targets in 3D for arbitrary diagnostic mounting with respect to the robot. The approach is able to avoid obstacles and avoid occlusion of the target by said obstacles. This can be used as part of autonomous investigation and characterisation performed by mobile robots in hazardous environments.
\end{abstract}

\section{INTRODUCTION}

Characterisation of materials is vital in the decommissioning of nuclear facilities to correctly categorise waste and sentence appropriately [1], [2], [3]. Typically, samples are retrieved manually by a human wearing a bulky and cumbersome air-fed suit and analysed ex-situ in a lab environment, however, there are inherent risks involved with humans entering active environments, along with producing extraneous waste such as suits and tools that have to be safely disposed of. Furthermore, with large sites it may not be feasible for a worker to retrieve the number of samples required for effective characterisation, whilst access to some locations is so restricted for safety reasons that sample collection may be precluded completely.

A robotic platform equipped with a compact scientific payload can manoeuvre through a inspection site to a target of interest and sample in-situ, without the need for human entry nor materials being removed. Laser Induced Breakdown Spectroscopy (LIBS) offers information on the elemental composition of materials and has been demonstrated as a viable technique to characterise nuclear materials [4], [5] as well as being deployed on robotic platforms such as the Mars Science Laboratory Rover, Curiosity [6].

*This work was supported by EPSRC Project EP/P018505/1

A. West, S. Watson and B. Lennox are with the School of Electrical and Electronic Engineering, The University of Manchester, Manchester, M13 9PL, UK andrew.west@manchester.ac.uk
Radiation dose is a known limit to the integrity of onboard electronics [7], [8], [9]. For a robot to minimise its received radiation dose (and maximise operational runtime), it may have to avoid certain locations where dose rate is high, furthermore, chemical and radiological contamination on surfaces also limit where a robot can traverse as it is counter-productive to knowingly spread already present contamination. Sensor placement by the robotic platform should be optimised to provide the best data quality possible in the minimum number of samples to maximise coverage of the site, whilst actively avoiding undesirable locations to prolong deployment lifetime.

This article presents a probabilistic approach to generate robot poses which optimise data quality for robot deployed focussed laser diagnostics, such as LIBS, sampling targets in $3 \mathrm{D}$.

\section{Robotic LIBS DEPLOYMENT}

Laser Induced Breakdown Spectroscopy uses focussed laser pulses to ablate a small volume of material and form a plasma. The optical emission from the plasma can then be used to infer the elemental composition of the material. The proposed LIBS system to be deployed uses a single lens arrangement for both laser pulse focus and plasma emission collection, and in this sense the diagnostic can be approximated as a point focus at some defined distance away from its origin on the robot.

The focal length is the first restriction in terms of placement of the LIBS diagnostic with respect to a target surface, being out of focus may lead to poor plasma formation and poor emission collection [10].

The spectral emission collected is also a function of angle of incidence of the LIBS diagnostic [11], and it is desirable to have the diagnostic as incident normal to the target surface as possible. This is a second restriction on where the diagnostic should be placed relative to the sample target to maximise data quality.

These two factors distance $D$ and angle of incidence $\gamma$ begin to define a sensor based approach to maximising data quality for fixed-focus point diagnostics.

As shown in Figure 1, the LIBS diagnostic transmission and collection optics are mounted on a pan and tilt stage onto a small Unmanned Ground Vehicle (UGV), approximated as holonomic. To simplify communicating the approach used in this investigation, the diagnostic is able to move about a singular point where the pan and tilt axis are co-located, and the joint is placed at a known location relative to origin of the chassis of the robot. For a given robot pose in Cartesian 
space $x y$ with yaw $\psi$ there are values for pan $\phi$ and tilt $\theta$ which result in the laser path intersecting at the target.

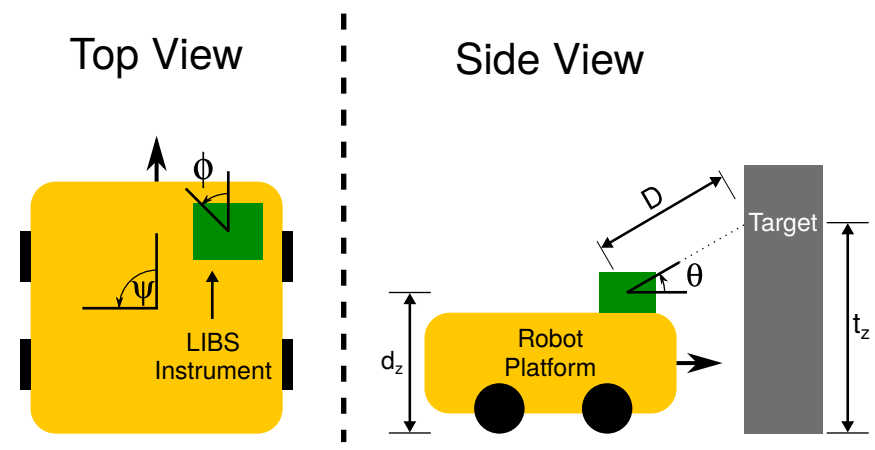

Fig. 1. Diagram of robot deployed LIBS. The LIBS instrument (green) may be located anywhere on the chassis of the robot.

There are restrictions on both pan and tilt axis due to physical and optical limits, for example the robot chassis may occlude the field of view when the diagnostic is tilted downwards, furthermore, the rest of the diagnostic (laser system, spectrometer) will likely be placed behind the collection optics at the rear of the robot, leading to occlusion at extreme angles of $\phi$.

Restrictions on $\phi$ and $\theta$ play a role in defining the optimum pose of the robot platform, however, other environmental factors need to be considered. Not only do physical obstacles limit the space in which the robot can be located, but may occlude the target for the diagnostic. Abstract obstacles in the form of radiation dose limits and contamination further restrict available robot poses.

\section{Optimising LiBS Diagnostic Placement}

A target is selected by an operator or by an autonomous data collection algorithm in 3D with reference to an a priori map, either collected by the robot as part of the operation or sourced elsewhere. A map created by the robot would be generated using techniques such as LiDAR. Using Simultaneous Localisation and Mapping (SLAM) and available path planning algorithms the robot is capable of navigating to the target. A well informed final pose of the robot can greatly increase data quality during sampling as discussed earlier.

$$
\begin{gathered}
D=f_{D}(x, y, \psi, t)=\left|\mathbf{d}_{x y z}-\mathbf{t}_{x y z}\right| \\
\gamma=f_{\gamma}(x, y, \psi, t)=\arccos \left(\frac{\mathbf{t}_{r p y} \cdot(\mathbf{d}-\mathbf{t})}{\left|\mathbf{t}_{r p y}\right||\mathbf{d}-\mathbf{t}|}\right) \\
\phi=f_{\phi}(x, y, \psi, t)=\arctan \left(\frac{\mathbf{t}_{y}-\mathbf{d}_{y}}{\mathbf{t}_{x}-\mathbf{d}_{x}}\right)-\psi \\
\theta=f_{\theta}(x, y, \psi, t)=\arccos \left(\frac{\mathbf{t}_{z}-\mathbf{d}_{z}}{\left|\mathbf{d}_{x y z}-\mathbf{t}_{x y z}\right|}\right)-\frac{\pi}{2}
\end{gathered}
$$

In a global frame of reference (most likely the coordinate frame of the map) and given $x y \psi$ values, vectors based on robot pose, a known target location $\mathbf{t}_{x y z}$ and orientation $\mathbf{t}_{r p y}$ and diagnostic location $\mathbf{d}_{x y z}$ and orientation $\mathbf{d}_{r p y}$ (as long as the relative transformation between the diagnostic and the robot is known), yields the ability to compute the factors $D \gamma \phi \theta$. These can be written as functions of $x y \psi$ as given in (1) - (4).

For a given target location and robot pose (as estimated through SLAM) in Euclidean space, the distance $D$ between the target $t$ and diagnostic $d$ in a global frame of reference can be calculated using simple vector mathematics $D=$ $\left|\left[t_{x}, t_{y}, t_{z}\right]-\left[d_{x}, d_{y}, d_{z}\right]\right|$.

As a general rule, in a lab environment the focal distance is kept as short as possible in the range of centimetres to tens of centimetres to increase the laser shot intensity (as focal spot size increases with focal distance) and improve the performance of the optical emission collection by increasing the numerical aperture, but far enough away that ablated material does not coat the optics too quickly.

In contrast to lab based LIBS instruments, robot deployed LIBS as part of the ChemCam instrument on the Curiosity rover operates with a focal distance between $1.3 \mathrm{~m}$ and $7 \mathrm{~m}$ as manoeuvring the rover is difficult and time consuming [6]. A terrestrial deployed robot does not suffer from the same unique challenges as interplanetary robots, and therefore can relocate more quickly and with less risk. A suitable standoff LIBS instrument designed to replace a human operator should be able to mimic the sampling volume of a human using a handheld LIBS instrument [12], being capable of sampling from $t_{z}=0 \mathrm{~m}$ to $t_{z}=1.9 \mathrm{~m}$. For a value of $t_{z}=1.9 \mathrm{~m}, d_{z}=0.5 \mathrm{~m}$ and $\theta=0.25 \pi$, the focal distance is approximately $2 \mathrm{~m}$. This focal distance of $2 \mathrm{~m}$ also allows not only for good diagnostic coverage, but when sampling hazardous substances the robot can be kept at a reasonable separation distance between the target and robot, greater than 4 times the robot radius for this configuration.

The depth of laser focus is a function of many experimental factors such as wavelength, beam profile and transmission optics, however, it is typically on the order of hundreds of micrometers. This precision positioning can not be readily achieved by a UGV, and so the instrument requires a variable focus mechanism to autofocus on length scales similar to the resultant error in LiDAR and wheel odometry SLAM.

A distance to a target estimated from a LiDAR map will have an error relative to the global frame of reference. Assuming an error of $\pm 30 \mathrm{~mm}$ [13], a robot that relies on a LiDAR system should have an autofocus system capable of focussing through this error range.

If the target normal is expressed as a vector (possibly derived from another coordinate system such as Euler angles or quaternions) the angle deviation of the diagnostic relative to the target normal can be calculated to find $\gamma$ in 3D using vector trigonometry.

The intensity of spectral emission collected is a function of the angle of incidence of the collection optics relative to the surface normal [14], and it is desirable to have the diagnostic as incident normal to the target surface as possible with a maximum angle of around $50^{\circ}(\approx 0.9 \mathrm{rad})$ where emission intensity is at a minimum. It is assumed that surfaces are either flat (such as walls) or convex (such as barrels and pipes), and so angles less than $\pi / 4$ rad are acceptable. 
The total orientation of the diagnostic required to point at the target then provides the required pan and tilt angles relative to the frame of the robot, once again if the transform between the robot and the diagnostic is known. The combination of the angle between the diagnostic and the target, minus the yaw of the robot to gives a $\phi$ value relative to the robot forward position. Rather than $\theta$ being relative to the $z$-axis, it is redefined to the $x y$ plane by subtracting $0.5 \pi$.

Each factor that determines data quality and the diagnostic's physical limitations can be described by a probability distribution, where the maximum value of 1 represents the best data quality associated with that parameter.

As error distance to the target can be assumed to be normally distributed, therefore, data quality in terms of distance can be modelled as a Gaussian centred at the focal distance $(\mu=2 \mathrm{~m})$, with a Full Width at Half Maximum (FWHM) equivalent to the error of $0.03 \mathrm{~m}$ in estimated distance to the target $(\sigma=2 * 0.03 /(2 \sqrt{2 \ln 2}))$.

The shape of emission intensity as a function of angle of incidence of the collection optical path can be approximated as a Gaussian [14]. At an angle of 0.9 rad the intensity is around 20\% [14], and so the Full Width at Fifth of Maximum is $2 * 0.9$, with the distribution centred about 0 $(\mu=0 \mathrm{rad}, \sigma=2 * 0.9 /(2 \sqrt{2 \ln 5}))$. The distribution tends towards zero for angles approaching $0.5 \pi$ to account for the sampling geometry. The distributions for $D$ and $\gamma$ are shown in Figure 2.
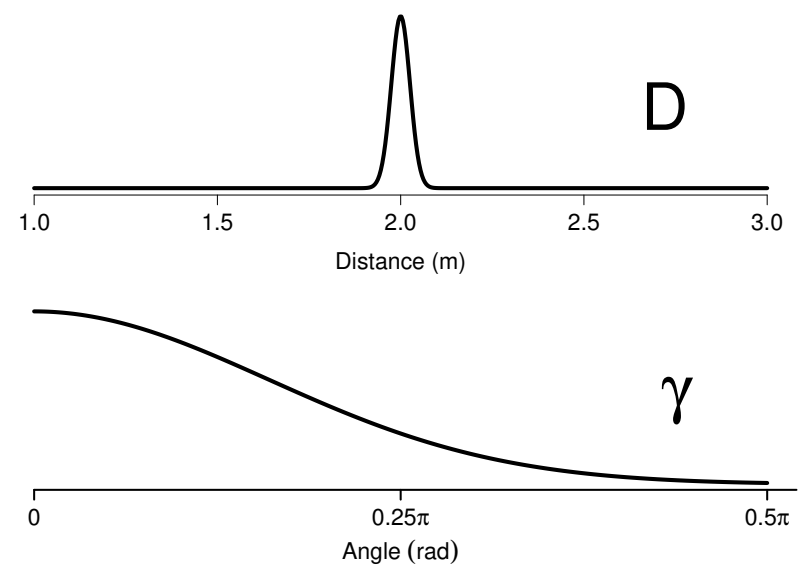

Fig. 2. Distribution of acceptable values for Distance $D$ and Angle of Incidence $\gamma$ to maximise data quality.

For $\phi$ and $\theta$, it is assumed that the laser and spectrometer will be mounted behind the pan and tilt mount of the LIBS diagnostic meaning that the pan axis should be restricted to $\pm 0.5 \pi$ to avoid occlusion. Occlusion of the robot chassis in the tilt axis restricts movement when targeting low $z$ height targets, there is the possibility that to maximise payload capacity a shelf would be installed above the LIBS instrument which would occlude targets at high $z$ height, however, this only requires consideration for a physical implementation. In place of constraints due to physical occlusion a value of $\pm 0.25 \pi \mathrm{rad}$ is used.

From a data quality perspective and occlusion perspective both $\phi$ and $\theta$ can be expressed as Super-Gaussian functions to approximate a uniform function whilst remaining continuous, and are centred around angles which represent the diagnostic directed in the forward direction of the robot. It is preferable for the diagnostic to be orientated in the robot forward position, this is commonly where other sensors such as obstacle avoidance and mapping LiDAR may be placed, as well as cameras for operator vision and sensors monitoring radiological contamination. Obstacle avoidance and avoidance of contamination is a clear benefit when traversing unstructured environments, whereas visual information about the plasma emission as seen through an RGB camera can be beneficial to a trained operator. The use of a higher-order Gaussian function still biases values around the mean (at robot forward), whereas a uniform distribution does not. A Super-Gaussian increases the power inside the exponent of a traditional Gaussian by a factor $p, \exp \left(-0.5((x-\mu) / \sigma)^{2 p}\right)$. Figure 3 shows pan $\phi$ is limited to a FWHM $\pm 0.5 \pi$ and tilt $\theta$ is limited to FWHM \pm 0.9 , with both using a factor $p=3$. Note that the FWHM is modified for a higher-order Gaussian where FWHM $=2 \sqrt[2 p]{2 \ln 2}$.
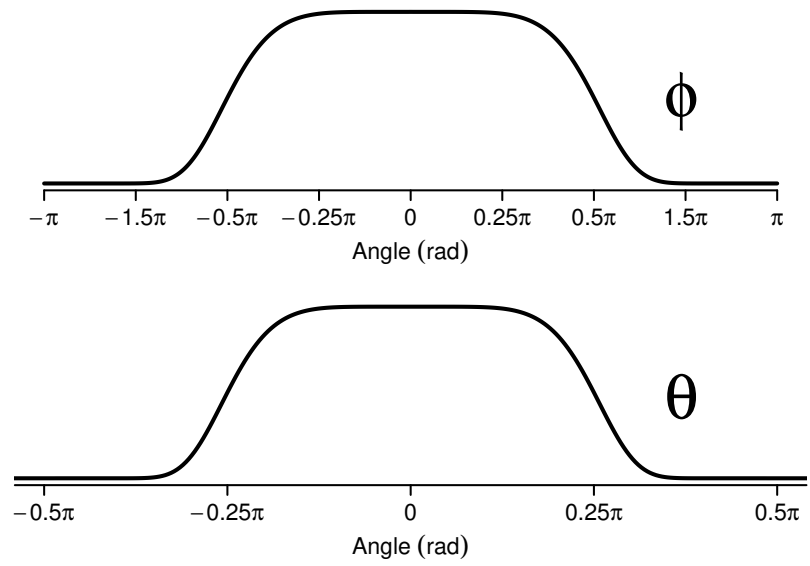

Fig. 3. Distribution of acceptable orientations of the diagnostic in Pan $\phi$ and Tilt $\theta$ axis.

$$
\begin{gathered}
P_{D}=g_{D}\left(\mu_{D}, \sigma_{D}, D\right) \\
P_{\gamma}=g_{\gamma}\left(\mu_{\gamma}, \sigma_{\gamma}, \gamma\right) \\
P_{\phi}=g_{\phi}\left(\mu_{\phi}, \sigma_{\phi}, \phi\right) \\
P_{\theta}=g_{\theta}\left(\mu_{\theta}, \sigma_{\theta}, \theta\right)
\end{gathered}
$$

Once all the factors are computed, the probability density is evaluated for each ((5)-(8) and the joint probability density computed by taking the product over all factors. The overall cost associated with these can instead be computed in log space to find overall probability density for a particular robot pose. By computing in log space, this reduces the arithmetic complexity as the product becomes a sum but also prevents numerical instabilities. Over all factors $i$ i.e. $D \gamma \phi \theta$, the overall cost $J$ is given in (9).

$$
J=\sum_{i} \log \left(P_{i}\right)
$$


To demonstrate how the cost function determines the optimum robot pose, the diagnostic is given an optimum focal length of $2 \mathrm{~m}$, mounted at the origin of the robot with a given vertical offset $z$. The target is placed at $(0,0, z)$ with normal vector $[0,-1,0]$ in global space. Using a height $z$ to match the diagnostic means net distance can be viewed in the $x y$ plane more intuitively. The colour values represent probability density, with red being best and blue being worst across the $x y$ space. To aid in visual representation of optimum robot pose, $J$ has been marginalised over $\psi$, i.e. it is the optimum pose integrated over all possible yaw angles for each $x y$ pair for this simple case.

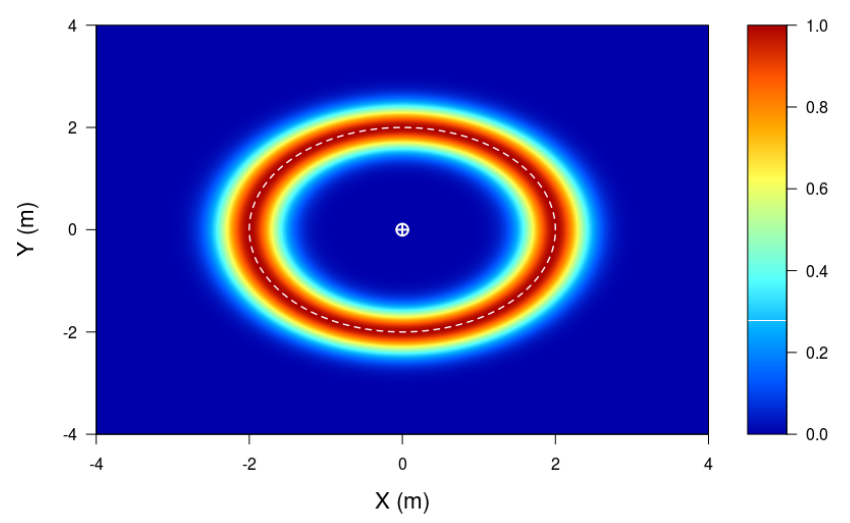

Fig. 4. Optimum positions around target centred at $(0,0)$ for only Distance as a constraint. The white dashed line indicates $D=2 \mathrm{~m}$.

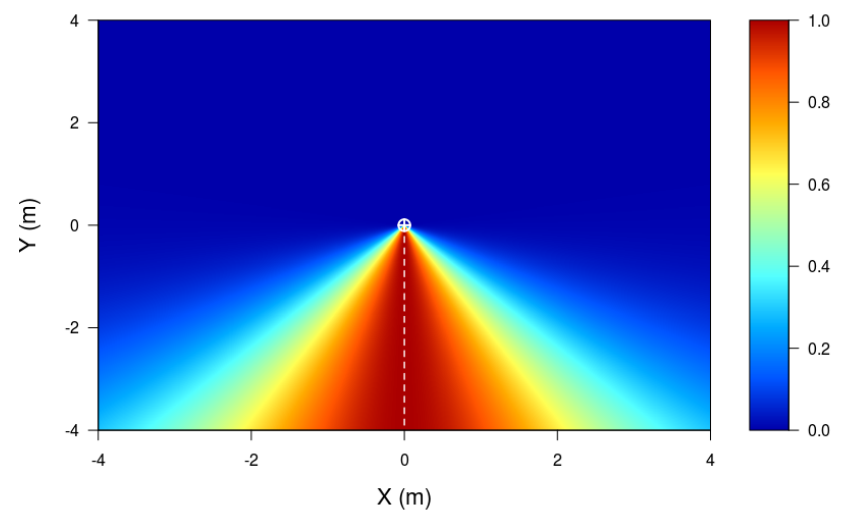

Fig. 5. Optimum positions around target centred at $(0,0)$ for only Angle of Incidence as a constraint. The white dashed line indicates $\gamma=0 \mathrm{rad}$.

In Figure 4, the constraint of distance unsurprisingly yields a radius of $2 \mathrm{~m}$ around the target for robot pose. Figure 5 shows angle of incidence prefers a cone centred around the target normal for possible robot poses. When the joint distribution for both $D$ and $\gamma$ is taken, optimum robot pose space becomes far more constrained to locations normal to the target, but still at a distance of $2 \mathrm{~m}$. This is demonstrated in Figure 6.

Currently, full maps have been produced to demonstrate how the probability space changes under different parameters for a given target, however, though the computation of the cost function is rapid it is still inefficient to compute over

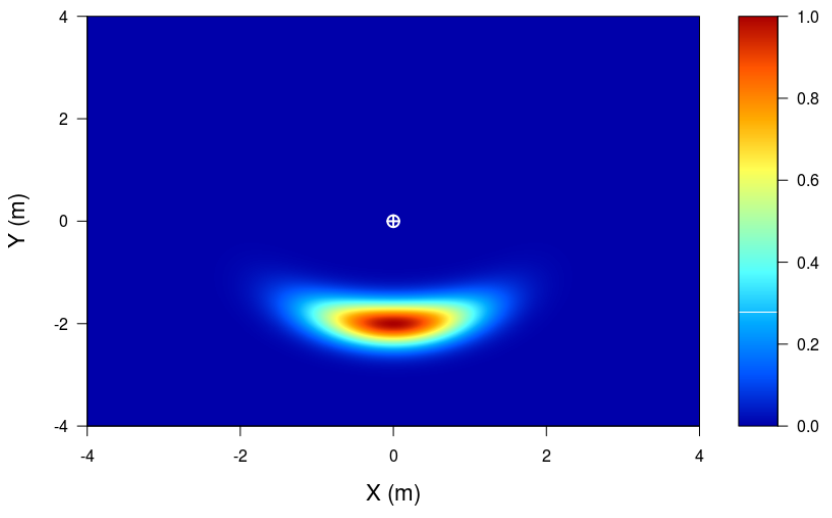

Fig. 6. Optimum placement to sample a target centred at $(0,0)$ to maximise data quality for Distance $D$ and Angle of Incidence $\gamma$.

all three dimensions of $x y \psi$ in high resolution and find the global optimum. Instead, an global optimiser can be used to traverse the probability space and find the optimum values for the robot pose as expressed with constraints in (10).

$$
\arg \max _{x, y, \psi}(J(x, y, \psi))
$$

$$
\begin{aligned}
\text { subject to: }-1.5 D \leq x \leq 1.5 D \\
-1.5 D \leq y \leq 1.5 D \\
0 \leq \psi \leq 2 \pi
\end{aligned}
$$

Robot yaw is simply defined as $0-2 \pi$, whereas the constraints for minimum and maximum values in $x$ and $y$ must be chosen based on the size of the available occupancy grid, but also the reasonable distances that the diagnostic can cover. For a focal distance of $2 \mathrm{~m}$ the maximum possible distance away from a target will never be greater than $2 \mathrm{~m}$. Assuming $\sigma_{D} \ll D$, to allow for solutions which are beyond the best focal distance an additional factor of $50 \%$ is included to give limits of $\pm 1.5 \mathrm{D}$. Optimisation was tested for simple scenarios where a solution is known such as the one shown in Figure 6, and reported expected values. The optimiser computed the result in less than 1 second, many orders of magnitude faster than brute force. This speed is beneficial in a deployed system where operational runtime is critical.

In Figure 4 there are values in front and behind the target which are optimal, however, the target is on a surface with the robot being physically incapable of sampling from some orientations, e.g. inside the surface.

Common implementations of SLAM and path planning utilise a 2D occupancy map to designate regions of space that contain obstacles [15]. If these maps are available, they can be used to check if a robot pose is permissible.

This obstacle map is used to disregard robot poses that fall within an obstacle on the occupancy grid. The map is taken in $x y$ configuration space, with an inscribed safety zone the same width as the robot radius around all obstacles, firstly to robustly avoid obstacles in hazardous environments and secondly to make integration into path planning algorithms more convenient. 
Obstacles not only prohibit where the robot can be located, but may occlude the target if it is between the diagnostic and the target. Using a 2D gridded occupancy map (this methodology can be scaled to 3D), a line drawing algorithm predicts the view path the diagnostic will encounter in a given pose.

To ensure this occlusion check is robust, the Supercover algorithm [16] is used rather than a more traditional Bresenham type algorithm [17] as the latter can miss some grid cells which are only partially intersected. The use of the $\mathrm{Su}-$ percover algorithm ensures every grid cell on the occupancy map that is intersected by the diagnostic is accounted for, as even partial occlusion will impact data quality.

Optimisation now must consider if the view path intersects with a region of the map that is designated an obstacle, then that robot pose should be disregarded. View paths that only intersect with the inscribed safety margin around obstacles or free space are unmodified, and the cost function is evaluated as normal.

Figure 7 shows a map in configuration space, where obstacles or unknown space are highlighted in red, and the inscribed safety margin in orange is equal to the radius of the robot. Any space in which the robot is free to move is given by light blue. Areas that would lead to occlusion of the target are represented with hashing.

To account for if a pose is legal or not the penalty function method is used [18]. If an $x y \psi$ pose falls in a forbidden or occluded location, then the joint distribution is penalised by a large negative value (in log space) which is unfavourable compared to the rest of the space (if Occluded $J=-1 \times$ $10^{6}$, else $\left.J=J(x, y, \psi)\right)$. This keeps the probability space continuous, however, it does introduce large gradients.

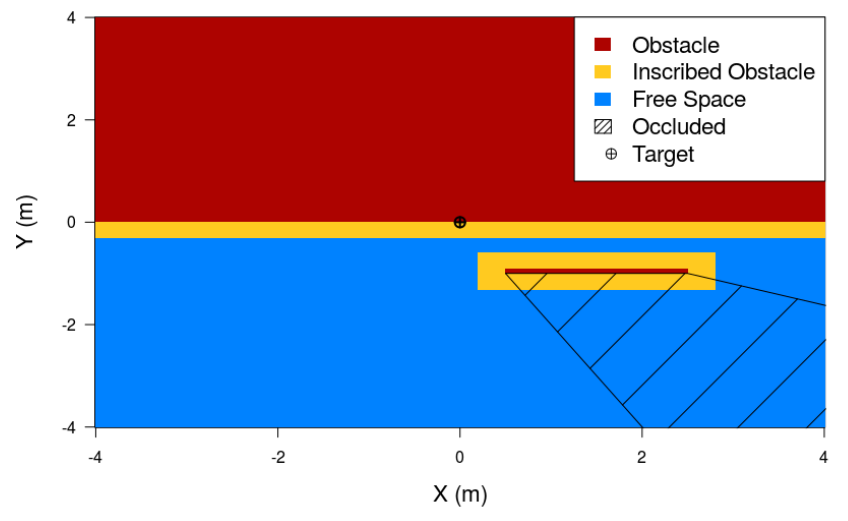

Fig. 7. 2D obstacle map.

As steep gradients may exist in the probability space due to the heavy penalisation for obstacles, occlusion, and radiation/contamination in log probability space, the Differential Evolution (DE) optimiser method was used as it is indifferent to gradients [19], however, there are many alternatives for local and global optimisation available.

The optimum poses found in Figure 6, are recomputed with the new information from the occlusion and obstacle map. This leads to a section of previously acceptable loca- tions to be excluded.

This use of an approximately binary, go/no-go cost for obstacles and occlusion is not suitable for threats such as radiation or contamination. No occlusion results from the presence of these threats, but the robot should still not enter these areas. For contamination, these locations can be treated similarly to a physical obstacle with some modification.

Radiation can be decoupled into two factors, the first is an unacceptable dose location where it would be treated the same as a physical obstacle i.e. forbidden, the second is the $1 / r^{2}$ dependence of the radiation dose away from a point source. It is preferable for the robot to avoid radiation exposure, however, low dose rates may be acceptable if there is a pay off in terms of data quality. A second occupancy map can be generated a priori which has contamination and high radiation locations treated as obstacles with an inscribed safety distance, but the map then has a decay as a function of distance from the source. For this study, an exponential decay is used instead of $1 / r^{2}$ as it is more simple to compute the joint distribution but more importantly it is much easier to tune the rate of decay around the target. This exponential weighting for radiation sources can be empirically defined by an operator to suit how cautious they wish to be compared to a more rigid $1 / r^{2}$ relationship.

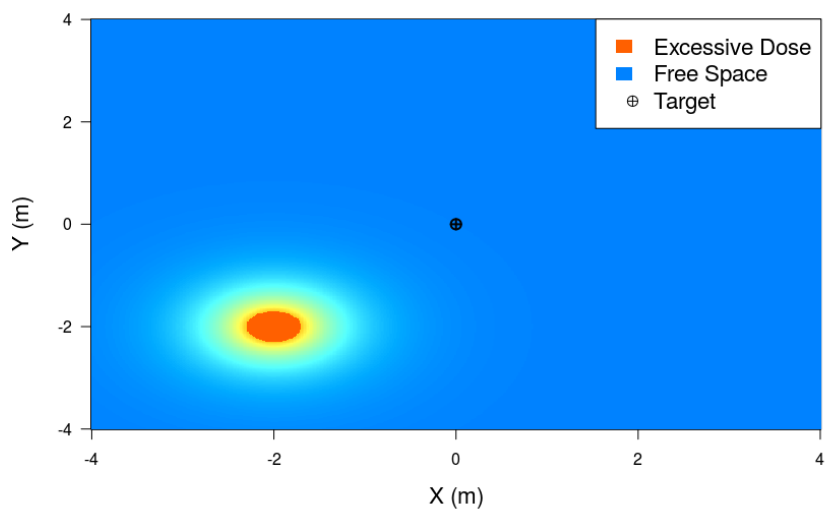

Fig. 8. Map containing a region of undesirable radiation dose, which decays as a function of distance away from a point source.

Figure 8 shows a region of unacceptable radiation dose from a point source at $(-2,-2)$, surrounded by an exponential decay representing the increased cost associated with a robot pose approaching the radiation source. Figure 9 shows optimisation for the $\psi=0$ case when combining obstacle occupancy, radiation/contamination occupancy and data quality for a target located at $(0,0)$. It can be observed that due to the presence of radiation, preferred robot poses are skewed to the $+x$ direction, and where obstacles are present or cause occlusion these are undesirable locations and accordingly the weighting is low compared to Figure 6.

Finally, the diagnostic was placed at the top right corner of the robot $(0.15,0.15)$ as shown in Figure 1, and the height of the target placed $1.5 \mathrm{~m}$ above the diagnostic mount and moved in the $-x$ direction closer to the influence of the radiation, $t=[-1,0, z+1.5]$. The extra height of the target 


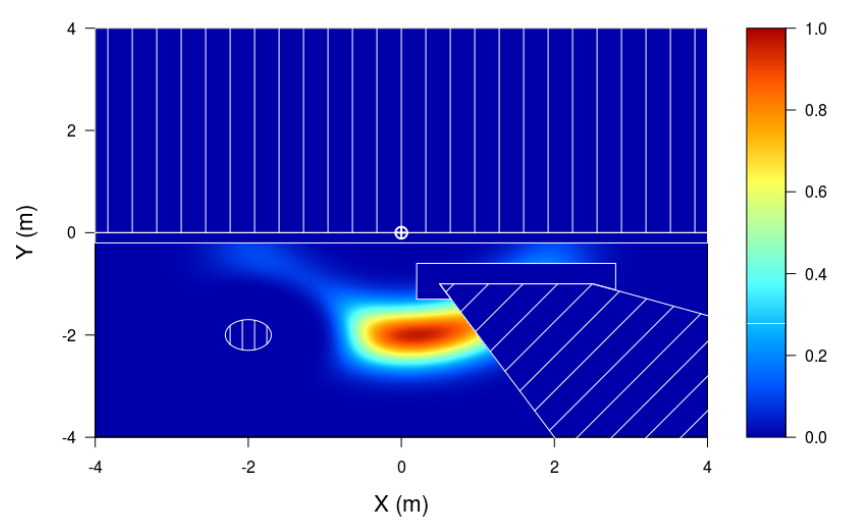

Fig. 9. Distribution of optimum sensor placement with occluding and non-occluding obstacles such as radiation.

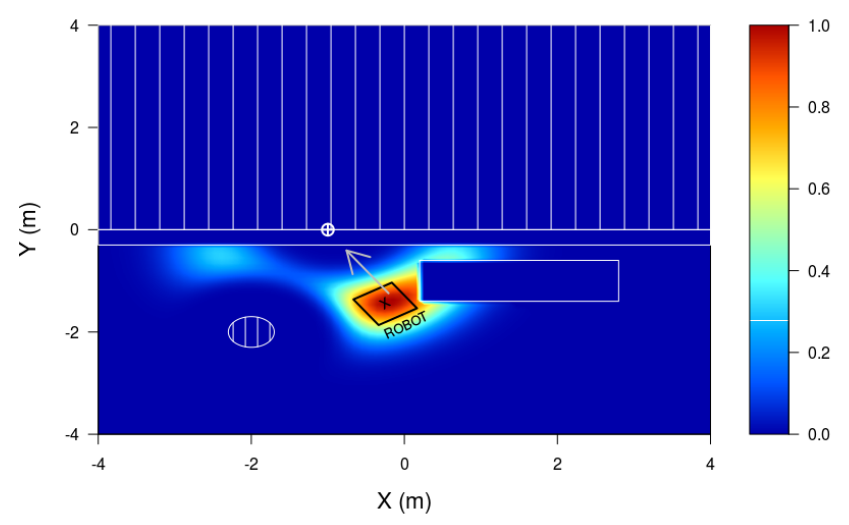

Fig. 10. Estimated best pose for a target placed at $(-1,0,1.5)$ with an off centre diagnostic position on robot. The black square represents the robot and the grey arrow represents the LIBS diagnostic.

impacts the angle of incidence to the target, and replicates the scenario shown in Figure 1. Figure 10 shows the robot position and orientation as found by the optimiser as a black box superimposed on the probability map in $x y$ associated with the optimum yaw angle. The diagnostic location and pointing vector to the target is in grey.

Though it would be beneficial for the robot to be placed further in the $-x$ direction to reduce the angle of incidence, the influence of the radiation source leads to the best robot pose to be at roughly $2 \mathrm{~m}$ distance but at a more extreme angle.

\section{CONCLUSIONS}

This sensor data quality based probabilistic approach to find robot poses for fixed-focus laser diagnostics is capable of determining optimum robot poses in $x y \psi$ for environments that include obstacles, occlusion and hazards such as radiation or chemical contamination. The use of continuous probability distributions for the various data quality factors allows for rapid estimation of poses through the use of a global optimiser. Using occupancy maps to for obstacles it is possible to disregard poses which would lead to a robot collision. Using a secondary or amalgamated occupancy map for radiation/contamination, it was possible to preference poses that are further from these sources, and therefore increase the operational time of the robot. Finally, it was shown that for a fixed-focus laser diagnostic mounted on a pan and tilt stage arbitrarily on a holonomic robot, an optimum position could be found for an arbitrary target located in 3D, in an environment with both occluding obstacles and radiation.

\section{ACKNOWLEDGEMENTS}

The authors wish to thank Dr. Chris Bowman for his guidance on global optimisation methods.

\section{REFERENCES}

[1] Nuclear Decommissioning Authority, Nuclear Decommissioning Authority Strategy: Strategy Effective from April 2016. TSO, 2016.

[2] Wilson and Stevens, "Decommissioning planning for the joint european torus fusion reactor," in Waste Management Symposia, 2007.

[3] Rosanvallon, Torcy, Chon, and Dammann, "Waste management plans for iter," Fusion Engineering and Design, vol. 109, pp. 1442 - 1446, 2016, proceedings of the 12th International Symposium on Fusion Nuclear Technology-12 (ISFNT-12).

[4] Martin, Allman, Brice, Martin, and Andre, "Exploring laser-induced breakdown spectroscopy for nuclear materials analysis and in-situ applications," Spectrochimica Acta Part B: Atomic Spectroscopy, vol. 74-75, pp. 177-183, aug 2012.

[5] Lang, et al., "Analysis of contaminated nuclear plant steel by laserinduced breakdown spectroscopy," Journal of Hazardous Materials, vol. 345, pp. 114-122, mar 2018.

[6] Maurice, et al., "The chemcam instrument suite on the mars science laboratory (msl) rover: Science objectives and mast unit description," Space Science Reviews, vol. 170, no. 1, pp. 95-166, Sep 2012.

[7] Nancekievill, Watson, Green, and Lennox, "Radiation tolerance of commercial-off-the-shelf components deployed in an underground nuclear decommissioning embedded system," in 2016 IEEE Radiation Effects Data Workshop (REDW). IEEE, 2016.

[8] Sharp and Decrton, "Radiation tolerance of components and materials in nuclear robot applications," Reliability Engineering \& System Safety, vol. 53, no. 3, pp. 291 - 299, 1996, safety of Robotic Systems.

[9] Nagatani, et al., "Gamma-ray irradiation test of electric components of rescue mobile robot quince," in 2011 IEEE International Symposium on Safety, Security, and Rescue Robotics, Nov 2011, pp. 56-60.

[10] Zuhlke, Bruce, Anderson, Alexander, and Parigger, "A fundamental understanding of the dependence of the laser-induced breakdown spectroscopy (LIBS) signal strength on the complex focusing dynamics of femtosecond laser pulses on either side of the focus," Applied Spectroscopy, vol. 68, no. 9, pp. 1021-1029, sep 2014.

[11] Miziolek, Palleschi, and Schechter, Laser induced breakdown spectroscopy. Cambridge University Press, 2006.

[12] Connors, Somers, and Day, "Application of handheld laser-induced breakdown spectroscopy (LIBS) to geochemical analysis," Applied Spectroscopy, vol. 70, no. 5, pp. 810-815, may 2016.

[13] Okubo, Ye, and Borenstein, "Characterization of the hokuyo URG041x laser rangefinder for mobile robot obstacle negotiation," in Unmanned Systems Technology XI, Gerhart, Gage, and Shoemaker, Eds. SPIE, may 2009.

[14] Multari and Cremers, "A time-resolved imaging study of cr (i) emissions from a laser plasma formed on a sample at nonnormal incidence," IEEE Transactions on Plasma Science, vol. 24, no. 1, pp. 39-40, 1996.

[15] Thrun, Burgard, and Fox, Probabilistic Robotics (Intelligent Robotics and Autonomous Agents series). The MIT Press, 2005.

[16] Andres, Nehlig, and Françon, "Supercover of straight lines, planes and triangles," in Discrete Geometry for Computer Imagery. Springer Berlin Heidelberg, 1997, pp. 243-254.

[17] Bresenham, "Algorithm for computer control of a digital plotter," IBM Systems Journal, vol. 4, no. 1, pp. 25-30, 1965.

[18] Pillo and Grippo, "Exact penalty functions in constrained optimization," SIAM Journal on Control and Optimization, vol. 27, no. 6, pp. 1333-1360, nov 1989.

[19] Storn and Price, "Differential evolution a simple and efficient heuristic for global optimization over continuous spaces," Journal of Global Optimization, vol. 11 , no. 4, pp. 341-359, 1997. 